TITLE:

\title{
Platinum cocatalyst loaded on calcium titanate photocatalyst for water splitting in a flow of water vapor
}

$\operatorname{AUTHOR}(S)$ :

Yoshida, H.; Yamada, R.; Yoshida, T.

\section{CITATION:}

Yoshida, H. ...[et al]. Platinum cocatalyst loaded on calcium titanate photocatalyst for water splitting in a flow of water vapor. ChemSusChem 2019, 12(9): 1958-1965

\section{ISSUE DATE:}

2019-05-08

URL:

http://hdl.handle.net/2433/242993

\section{RIGHT:}

This is the peer reviewed version of the following article: H. Yoshida, R. Yamada, T. Yoshida, ChemSusChem 2019, 12 1958, which has been published in final form at https://doi.org/10.1002/cssc.201802799. This article may be used for non-commercial purposes in accordance with Wiley Terms and Conditions for Use of Self-Archived Versions.; The fulltext file will be made open to the public on 8 May 2020 in accordance with publisher's 'Terms and Conditions for SelfArchiving'.: This is not the published version. Please cite only the published version.: この論文は出版社版でありません 。引用の際には出版社版をご確認ざ利用ください。 


\title{
Platinum cocatalyst loaded on calcium titanate photocatalyst for water splitting in a flow of water vapor
}

\author{
Hisao Yoshida, ${ }^{[a, b, b]}$ Ryoko Yamada, ${ }^{[c]}$ and Tomoko Yoshida ${ }^{[c, d]}$
}

\begin{abstract}
Photocatalytic water splitting by using heterogeneous photocatalysts has been intensively studied in recent decades, where the use of suitable cocatalyst has been recognized as one of the key factors to enhance the photocatalytic activity. Although platinum is a representative cocatalyst for many heterogeneous photocatalytic reactions, it has not been used for photocatalytic water splitting due to its high catalytic activity for the reverse reaction of water splitting. In the present study, platinum nanoparticles as cocatalyst were loaded on calcium titanate photocatalyst by a conventional impregnation method and a photodeposition method, and examined for photocatalytic water splitting in a flow of water vapor. It was revealed that platinum nanoparticles loaded by the impregnation method preserve the oxidized form on calcium titanate photocatalyst even under photoirradiation and can well promote the hydrogen and oxygen production in the photocatalytic water vapor splitting without promoting the reverse reaction.
\end{abstract}

\section{Introduction}

The development of sustainable energy production is one of the most important subjects for the near future, and the utilization of solar energy is most desired. Solar energy is however unstorable as it is, so that it should be converted to chemical potential. Hydrogen is a storable and useful energy carrier with the high energy content and non-polluting nature. For example, it can be effectively converted into electricity by a fuel cell or into motive power by a hydrogen-fueled engine without any emission other than water. Thus, photocatalytic water splitting to produce hydrogen by using solar energy is an ideal method, and many studies have been intensively and widely carried out to develop highly active photocatalysts [1-4].

\footnotetext{
[a] Prof. H. Yoshida

Graduate School of Human and Environmental Studies

Kyoto University

Yoshida-nihonmatsu-cho, Sakyo-ku, Kyoto 606-8501 (Japan)

E-mail: yoshida.hisao.2a@kyoto-u.ac.jp

[b] Prof. H. Yoshida

Elements Strategy Initiative for Catalysts and Batteries (ESICB)

Kyoto University

Kyotodaigaku-Katsura, Nishikyo-ku, Kyoto 615-8520 (Japan)

[c] Prof. H. Yoshida, R. Yamada, Prof. T. Yoshida

Graduate School of Engineering

Nagoya University

Furo-cho, Chikusa-ku, Nagoya 464-8603 (Japan)

[d] Prof. T. Yoshida

Advanced Research Institute for Natural Science

Osaka City University

3-3-138, Sugimoto, Sumiyoshi-ku, Osaka 558-8585 (Japan)
}

Heterogeneous semiconductor photocatalysts such as metal oxide particles have been well studied. When the photocatalyst absorbs light, the photoenergy generates excited electrons and holes as charge carriers. If they spatially separate and reach the surface of the semiconductor particles without their recombination, they can react with molecules or ions to yield the products. Thus, decreased recombination of electrons and holes would increase the photocatalytic performance. Cocatalyst, such as metal or metal oxide nanoparticles, attached on the photocatalyst surface have often been employed as an electron receiver to promote the charge separation and reduce recombination. Since water splitting is a highly endergonic and thermodynamically unfavorable reaction and the reverse reaction can be easily promoted, cocatalyst for water splitting should be inactive for the reverse reaction. For water splitting, some kinds of metal oxide cocatalysts such as nickel oxide $(\mathrm{NiO})$, ruthenium oxide $\left(\mathrm{RuO}_{2}\right)$ and chromium oxide covered rhodium $\left(\mathrm{CrO}_{x} / \mathrm{Rh}\right)$ cocatalysts [1-3] have been representatively employed.

In contrast, platinum metal $(\mathrm{Pt})$ cocatalyst tends to be avoided for water splitting photocatalyst since it is a highly active catalyst for the reverse reaction of water splitting [5-7], i.e., the reaction between hydrogen and oxygen to produce water is promoted by the Pt cocatalyst. In the case of water splitting of water vapor in gas phase, these products can interact with the Pt surface more easily and react with each other $[5,6]$. In the case of water splitting in an aqueous solution, when the produced hydrogen and oxygen can immediately escape from the photocatalyst surface, they may scarcely come back to the Pt surface to react with each other. But, when the product gases make a surface bubble, they may interact with the Pt catalyst. Thus, heterogeneous photocatalysts for water splitting, especially for water vapor splitting, requires a cocatalyst that is catalytically inactive for the reverse reaction of water splitting. Here, it should be reminded that $\mathrm{Pt}$ is a representative and highly active cocatalyst for many photocatalytic reactions, including hydrogen production from methane and water [8-12] and various organic reactions with hydrogen formation [13-19], and the property of the Pt cocatalyst can be varied with the loading method and the resulting chemical state $[7,20]$. Thus, if it becomes inactive for the reverse reaction, the Pt cocatalyst may become an excellent cocatalyst.

Calcium titanate $\left(\mathrm{CaTiO}_{3}\right)$ is an inexpensive material consisting of ubiquitous elements. It has been already studied for some photocatalytic reactions such as steam reforming of methane to produce hydrogen [21,22], carbon dioxide splitting into carbon monoxide and oxygen [23,24], and also water splitting both in gaseous phase [21] and liquid phase [25]. Among them, it is notable that, $\mathrm{Pt}$ cocatalyst loaded on the $\mathrm{CaTiO}_{3}$ photocatalysts is available for the water splitting even in the gas phase [21]. 
In the present study, the photocatalytic activity of Pt loaded $\mathrm{CaTiO}_{3}$ (CTO) photocatalyst was examined for the water vapor splitting. Pt cocatalyst was loaded on СTO photocatalyst by two methods, a conventional impregnation method and a photodeposition method, which are referred to as $\mathrm{Pt}(x) /$ CTO-imp and $\mathrm{Pt}(x) / \mathrm{CTO}$-photo, respectively, where $x$ indicates the loading amount of Pt. The photocatalytic activity of the Pt/CTO samples were evaluated, and the structure and oxidation state of the $\mathrm{Pt}$ cocatalyst were investigated to understand the property of the $\mathrm{Pt}$ cocatalyst on the surface of $\mathrm{CaTiO}_{3}$ photocatalyst.

\section{Results and Discussion}

\section{Photocatalytic performances}

The photocatalytic water splitting was examined by using the $\mathrm{Pt}(0.01) / C T O-i m p$ and $\mathrm{Pt}(0.01) / C T O-p h o t o$ samples in a flow of water vapor. The time courses of the production rates were shown

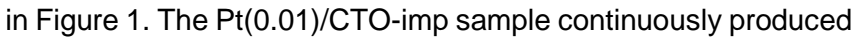
both hydrogen and oxygen after the induction period (Figures 1a and $1 b)$. This confirms that the $\mathrm{Pt}(0.01) / C T O-i m p$ sample can promote photocatalytic water splitting. Since bare CTO sample exhibited no activity for the water splitting, it was evidenced that the Pt cocatalyst can drastically enhance the photocatalytic activity for water vapor splitting.

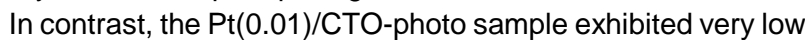
production rate of hydrogen (Fig. 1c) and no detectable formation of oxygen in the present experimental condition. As mentioned above, Pt cocatalyst is known to promote the reverse reaction of water splitting to decrease the production rate of hydrogen and oxygen [5-7], which is consistent with the present result.

Thus, it was confirmed that Pt cocatalyst on the CTO sample can promote both water splitting and the reverse reaction, and it is proposed that the $\mathrm{Pt}$ cocatalyst deposited on the $\mathrm{CaTiO}_{3}$ surface by the impregnation method can promote water splitting rather than the reverse reaction.

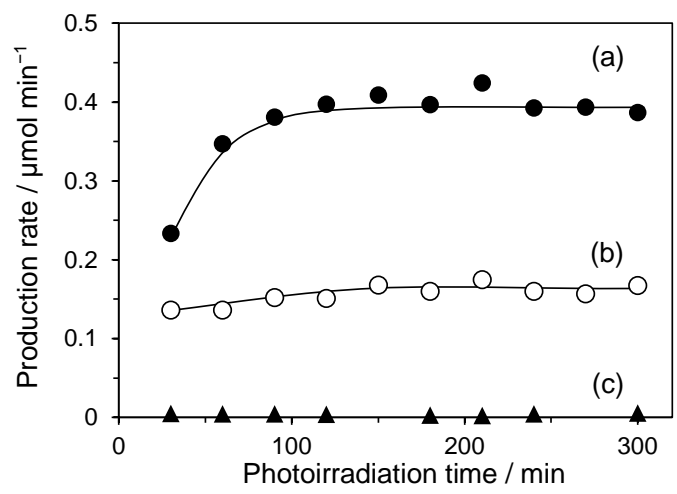

Figure 1. Time course of photocatalytic production rate of (a) $\mathrm{H}_{2}$ and (b) $\mathrm{O}_{2}$ with the $\mathrm{Pt}(0.01) / C T O$-imp sample, and (c) that of $\mathrm{H}_{2}$ with the $\mathrm{Pt}(0.01) / C T O-p h o t o$ sample in the flow of water vapor.
To elucidate the reason why the photocatalytic property was so different between the two samples, the catalytic reaction tests for the reverse reaction between hydrogen and oxygen were carried out with these samples without photoirradiation at $318 \mathrm{~K}$ in a closed reactor for $30 \mathrm{~min}$, where the reaction temperature was chosen to be similar to that for the present photocatalytic reaction test. The results are shown in Figure 2. In the presence of hydrogen and oxygen and the $\mathrm{Pt}(0.01) / C T O$-photo sample, the partial pressure of hydrogen clearly decreased with time (Figure $2 b)$. This means that the water formation is promoted by the Pt catalyst, which is consistent with literature [5-7]. In contrast, even in the presence of the $\mathrm{Pt}(0.01) / C T O-i m p$ sample, no decrease was observed at all (Figure 2a). This clearly indicates that the Pt species loaded on the $\mathrm{CaTiO}_{3}$ surface by the impregnation method exhibit no catalytic activity for the reaction between hydrogen and oxygen to produce water in dark, which should be one of the reasons why the $\mathrm{Pt}(0.01) / C T O$-imp sample exhibited high production rates in the water splitting (Figures $1 \mathrm{a}$ and $1 \mathrm{~b}$ ) in

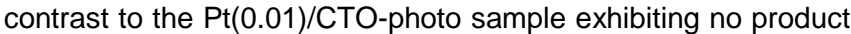
formation (Figure 1c). That is, the Pt species on the former sample can enhance the photocatalytic water splitting but cannot catalyze the reverse reaction in dark, while the Pt species on the latter sample cannot give the products of water splitting because it promotes preferably the reverse reaction more than the photocatalytic water splitting. Thus, the structure or chemical state of these Pt species would be different from each other, originating from the different loading methods on the same СТО sample.

In the present study, the exact Pt loading amount of the two samples, the $\mathrm{Pt}(0.01) / C T O-i m p$ and $\mathrm{Pt}(0.01) / C T O-p h o t o ~ s a m p l e s$, could not be determined by a spectroscopic method due to the very low concentration. However, even if the exact Pt loading amount are slightly different from the values expected from the amount of the used Pt precursor, the slight deviation of the loading amount would not influence the discussion hereinafter since the difference of the photocatalytic and catalytic properties shown in Figures 1 and 2 are very clear, i.e., the Pt(0.01)/CTO-imp sample exhibited the photocatalytic activity for the water splitting and no catalytic activity for the reverse reaction, while the Pt(0.01)/CTOphoto sample did not show the photocatalytic activity for water splitting but showed the catalytic activity for the reverse reaction in dark.

\section{Structure and property of the $\mathrm{CaTiO}_{3}$ photocatalyst}

Before going to the investigation of the Pt species, the structure and property of the CTO sample was clarified by some methods such as field emission scanning electron microscopy (SEM), powder X-ray diffraction (XRD), diffuse reflectance UV-visible spectrum, and $\mathrm{N}_{2}$ adsorption experiments. The SEM image presents that the CTO particles are roundish particles covered with some flat facets (Figure 3a). The XRD pattern shows that the crystal structure is assignable to $\mathrm{CaTiO}_{3}$ perovskite without any impurity phases (Figure 3b). The DR UV-vis spectrum of the CTO sample shows a large absorption band in the range less than 350 $\mathrm{nm}$ in wavelength (Figure 3c), which corresponds to the 
photoexcitation from the valence band to the conduction band of $\mathrm{CaTiO}_{3}$. Estimated bandgap was $3.4 \mathrm{eV}$. The spectrum also shows a small shoulder in the range of $350-400 \mathrm{~nm}$ in wavelength. Since no impurity phase was observed in the XRD profile, this shoulder would be assigned to crystal defects or very small $\mathrm{TiO}_{2}$ moiety. This photoabsoption feature is similar to that of the $\mathrm{CaTiO}_{3}$ particles prepared by a solid state reaction method in the previous study [21]. The $\mathrm{N}_{2}$ adsorption experiments revealed that the BET specific surface area was $1.62 \mathrm{~m}^{2} \mathrm{~g}^{-1}$.

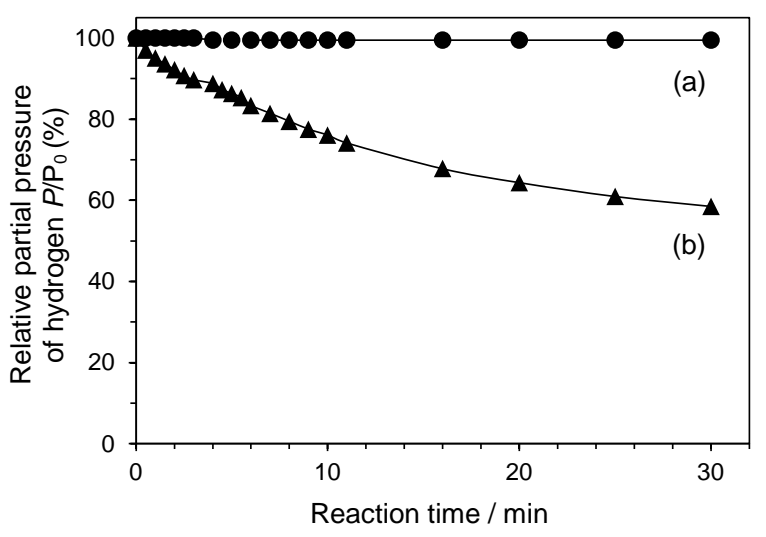

Figure 2. Time course of relative partial pressure of hydrogen in the reverse reaction of water splitting in dark with (a) the $\mathrm{Pt}(0.01) / \mathrm{CTO}$-imp sample and (b) the $\mathrm{Pt}(0.01) /$ CTO-photo sample. The reaction test was carried out in a closed reactor at $318 \mathrm{~K}$ in a reactor of $62 \mathrm{ml}$ without photoirradiation. Initial partial pressure of $\mathrm{H}_{2}$ and $\mathrm{O}_{2}$ were 3 and $1.5 \mathrm{kPa}$, respectively.
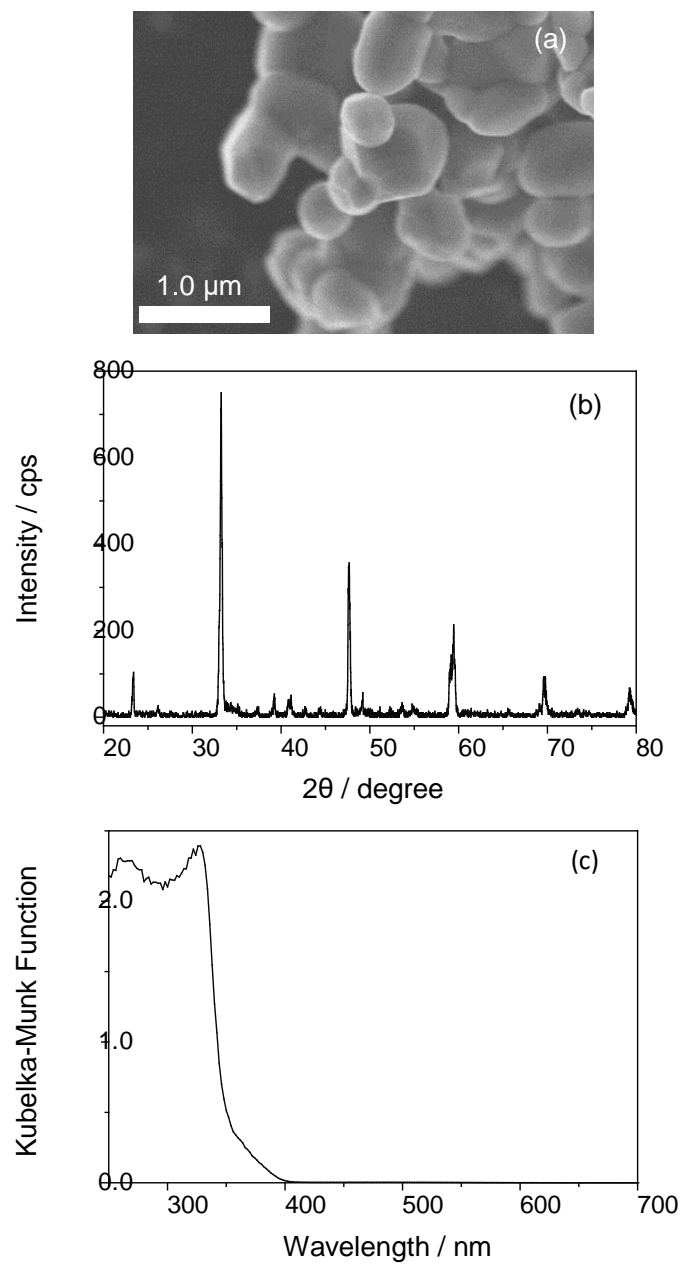

Figure 3. SEM image (a), XRD pattern (b), and DR UV-visible spectrum (c) of the prepared $\mathrm{CaTiO}_{3}$ sample.

\section{Structure and chemical state of the platinum species}

To understand the different properties of the Pt species on the two samples mentioned above, the structure and chemical state of the Pt species were investigated by transmission electron microscopy (TEM) and X-ray absorption fine structure (XAFS), where the Pt/CTO samples with larger amount of Pt such as 0.2 wt $\%$ were employed to obtain clear images and spectra. As clarified later, the difference of the loading amount employed here is not important factor to obtain the conclusion.

Figures $4 \mathrm{a}$ and $4 \mathrm{~b}$ show TEM images of the $\mathrm{Pt}(0.2) / C T O-i m p$ and $\mathrm{Pt}(0.2) / C T O-p h o t o$ samples, respectively. Pt nanoparticles are observed on each sample, confirming the Pt cocatalyst are deposited as nanoparticles on the CTO surface. The size distributions of these Pt nanoparticles were summarized in the Figures $5 \mathrm{a}$ and $5 \mathrm{~b}$. The size distributions were similar but slightly different on the $\mathrm{Pt}(0.2) / C T O-i m p$ and $\mathrm{Pt}(0.2) /$ CTO-photo samples, which were predominantly in the range of $1-2$ and $2-4 \mathrm{~nm}$ in diameter, respectively. 

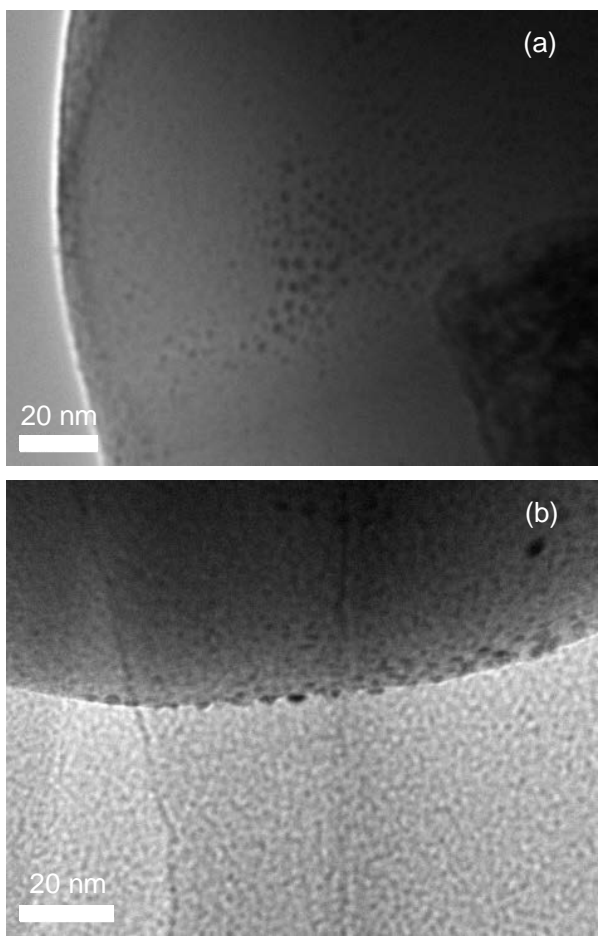

Figure 4. TEM images of (a) the $\mathrm{Pt}(0.2) / \mathrm{CTO}$-imp sample and (b) the $\mathrm{Pt}(0.2) /$ CTO-photo sample.
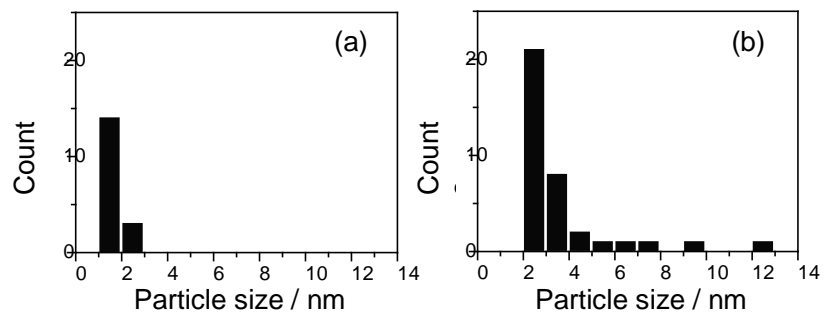

Figure 5. Pt particle size distributions on (a) the Pt(0.2)/CTO-imp sample and (b) the $\mathrm{Pt}(0.2) / C T O-p h o t o ~ s a m p l e$, which were estimated from TEM images.

To elucidate the chemical state of the Pt species, Pt LוI-edge XAFS spectra of these samples were recorded and analyzed. Figure 6 shows X-ray absorption near edge structure (XANES) of the samples in the following three steps, before the reaction test, after the pretreatment (photoirradiation in air), and after the reaction test, with the reference samples, $\mathrm{PtO}_{2}$ and $\mathrm{Pt}$. XANES spectra of the $\mathrm{Pt}(0.2) / C T O-i m p$ samples were similar to that of $\mathrm{PtO}_{2}$, indicating that the $\mathrm{Pt}$ species on the samples in these steps were in oxidized state similarly (Figures $6 \mathrm{Aa}, 6 \mathrm{Ab}$, and $6 \mathrm{Ac}$ ). On the other hand, the spectra of the $\mathrm{Pt}(0.2) / C T O-p h o t o ~ s a m p l e s$ clarified the variation of the state of the Pt species, i.e., although the photodeposited Pt nanoparticles were naturally oxidized in air before the pretreatment (Figure 6Ba), they were almost reduced by the photoirradiation even in air (Figure 6Bb), and they were still reduced after the photocatalytic reaction test (Figure $6 \mathrm{Bc}$ ). The Fourier-transformed extended X-ray absorption fine structures (EXAFS) of these samples support these results (Figure 7). The peak assignable to the $\mathrm{Pt}-\mathrm{O}$ shell are clearly observed around 1.6-1.7 $\AA$ for the Pt(0.2)/CTO-imp samples in the three steps and also for the $\mathrm{Pt}(0.2) / C T O-$ photo sample before the pretreatment The peaks assignable to the Pt-Pt shells around 2.3-2.9 $\AA$ appeared on the $\mathrm{Pt}(0.2) / C T O-p h o t o$ samples after the pretreatment and after the photocatalytic reaction test.

These results clearly show that the chemical state of the $\mathrm{Pt}$ species are quite different on the two samples, the Pt(0.2)/CTOimp sample and the $\mathrm{Pt}(0.2) / C T O-$ photo sample after the pretreatment and during the photocatalytic reaction, i.e., the $\mathrm{Pt}$ species loaded by the impregnation method on the $\mathrm{CaTiO}_{3}$ surface were in the oxidized state, while the Pt species loaded by the photodeposition method on the $\mathrm{CaTiO}_{3}$ surface were metallic nanoparticles. Thus, it was clarified that the oxidized Pt species can promote the photocatalytic water splitting as a cocatalyst on the $\mathrm{CaTiO}_{3}$ photocatalyst to produce hydrogen and oxygen due to the property of catalytically inactive for the reverse reaction. In contrary, it was evidenced that the metallic Pt nanoparticles cannot give enough products of the water splitting due to the catalytically active property for the reverse reaction. This is consistent with the reported matter that the oxidized and reduced Pt clusters are inactive and active for the hydrogen oxidation, respectively [7]. 


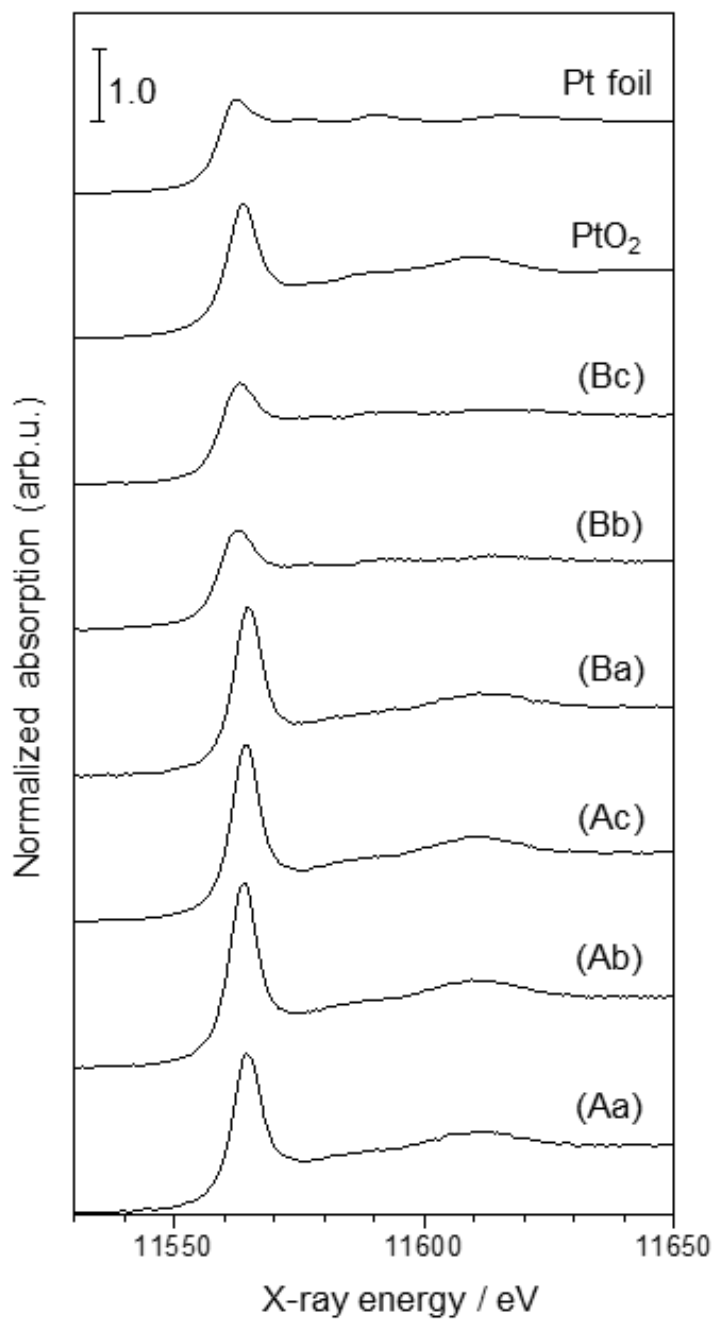

Figure 6. $\mathrm{Pt} \mathrm{L}_{\| 1}$-edge XANES spectra of $(\mathrm{A})$ the $\mathrm{Pt}(0.2) / \mathrm{CTO}$-imp sample and (B) the $\mathrm{Pt}(0.2) / C T O-p h o t o$ sample; (a) before the photocatalytic reaction test (b) after the pre-treatment, and (c) after the photocatalytic reaction test. The reference spectra of $\mathrm{PtO}_{2}$ and $\mathrm{Pt}$ foil are also shown.

The difference of the chemical state of the Pt species on these samples suggests that their initial states after the loading are also different from each other. It is reported that the platinum species supported on basic metal oxide are stabilized as an oxidized state and those supported on acidic metal oxide has oxidationresistance property to be in metallic state even under oxidative conditions [26,27]. From this property and the results in the present study, it is proposed that the $\mathrm{CaTiO}_{3}$ surface would have a basic property, which might originate from $\mathrm{CaO}$ moiety on the surface, and stabilize the Pt species as an oxidized form such as well-dispersed $\mathrm{Pt}$ oxo-anion $\mathrm{PtO}_{\alpha}{ }^{\delta-}[26]$, since for example $\mathrm{CaPt}_{3} \mathrm{O}_{4}$ has much higher thermal stability than $\mathrm{PtO}_{2}$ [28]. In the present impregnation method, the precursor $\mathrm{H}_{2} \mathrm{PtCl}_{6}$ would be adsorbed, dried, and calcined to be oxidized on the $\mathrm{CaTiO}_{3}$ surface, which would provide a well-dispersed and oxidized platinum particles or a kind of mixed oxide moieties with the surface of $\mathrm{CaTiO}_{3}$. Thus, it is suggested that the oxidized Pt species on the $\mathrm{CaTiO}_{3}$ surface would responsible for the production of hydrogen and oxygen in the photocatalytic water vapor splitting.

On the other hand, in the photodeposition method, the Pt species are reduced to be metallic nanoparticles. Although they would be once naturally oxidized by the air, the state would be different from that obtained in the impregnation method. They can be reduced by the additional photoirradiation.

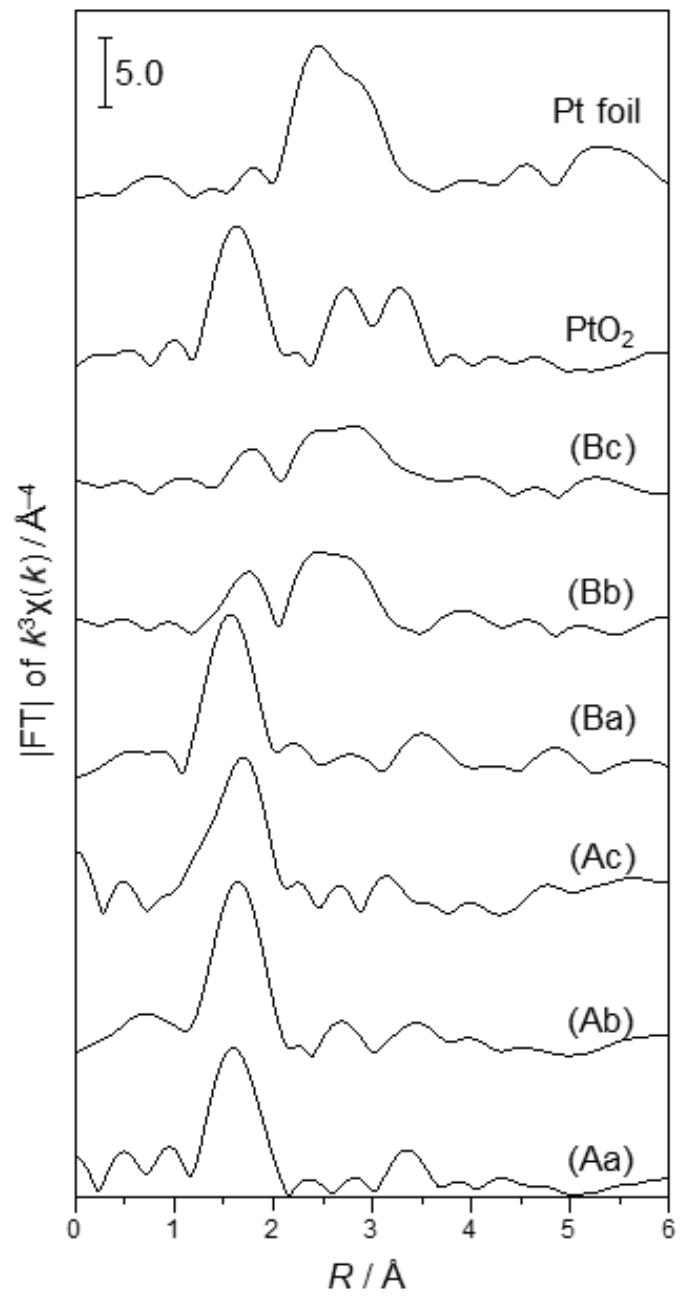

Figure 7. $\mathrm{Pt} \mathrm{L}_{\mathrm{III}}$-edge Fourier-transformed EXAFS spectra of $(\mathrm{A})$ the

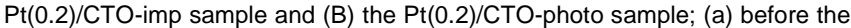
photocatalytic reaction test, (b) after the pre-treatment, and (c) after the photocatalytic reaction test. The spectra of $\mathrm{Pt}$ foil and $\mathrm{PtO}_{2}$ are also shown as the references.

\section{Post treatments}

The loading method was found to determine the chemical state and thus the catalytic property of the Pt species on the $\mathrm{CaTiO}_{3}$ surface. Furthermore, post treatments were examined as 
follows. The $\mathrm{Pt}(0.01) / C T O-p h o t o$ sample was oxidized in air at $773 \mathrm{~K}$ for $6 \mathrm{~h}$ (referred to as $\mathrm{Pt}(0.01) / \mathrm{CTO}-$ photo-ox). This oxidation treatment was expected to change the catalytic property of the Pt species and increase the formation rate of the products in water vapor splitting due to the decrease of the reverse reaction rate. As shown in Figure 8, the $\mathrm{Pt}(0.01)$ /CTO-photo-ox sample (Figure $8 \mathrm{~b}$ ) exhibited a higher hydrogen production rate in the photocatalytic water vapor splitting and a lower rate for the reverse reaction in dark than the original $\mathrm{Pt}(0.01) / C T O-p h o t o$ sample (Figure $8 \mathrm{~d}$ ). It is suggested that the oxidation treatment much suppressed the rate of the reverse reaction and thus the hydrogen production rate increased. However, the production rate

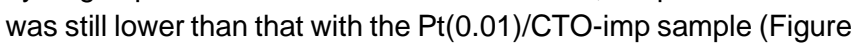
8a) although the two samples was finally oxidized at the same conditions, i.e., calcination at $773 \mathrm{~K}$ for $6 \mathrm{~h}$. This means that not only the final treatment condition but the history of the preparation is also important. As mentioned above, the impregnation method would once provide adsorbed platinum (IV) species on the $\mathrm{CaTiO}_{3}$ surface, and they would become well-dispersed oxidized species or a kind of mixed oxide moieties by the reaction with the $\mathrm{CaTiO}_{3}$ surface. In the photodeposition method, the precursor was reduced by the photoexcited electrons on the $\mathrm{CaTiO}_{3}$ surface to form metallic nanoparticles and then naturally oxidized by the air during storage. The additional calcination of this nanoparticles as a post treatment would give further oxidized species like as $\mathrm{PtO}_{2}$ nanoparticles, which would be somewhat different from the $\mathrm{Pt}$ species provided by the impregnation method and easily reduced to show the activity for the reverse reaction to some extent.

In an opposite manner, the $\mathrm{Pt}(0.01) / C T O-i m p$ sample containing the initially oxidized $\mathrm{Pt}$ species was reduced by hydrogen at $473 \mathrm{~K}$ for $30 \mathrm{~min}$ (referred to as $\mathrm{Pt}(0.01) / C T O-i m p-$ red). This sample exhibited a much lower hydrogen production rate and a higher activity for the reverse reaction (Figure $8 \mathrm{c}$ ) than the original one (Figure 8a). However, this sample exhibited higher hydrogen production than the $\mathrm{Pt}(0.01) / C T O-p h o t o ~ s a m p l e$, suggesting that the final chemical state of the Pt species would be different from that of the Pt(0.01)/CTO-photo sample, i.e., the oxidized Pt species dispersed on the СTO surface would not be fully reduced or maintain their high dispersion.

As shown in Figure 8, the four samples exhibit a clear relationship between the photocatalytic hydrogen production rate and the progress of the reverse reaction, i.e., the Pt species having less catalytic activity for the reverse reaction exhibited higher production rate in the photocatalytic water vapor splitting as expected.

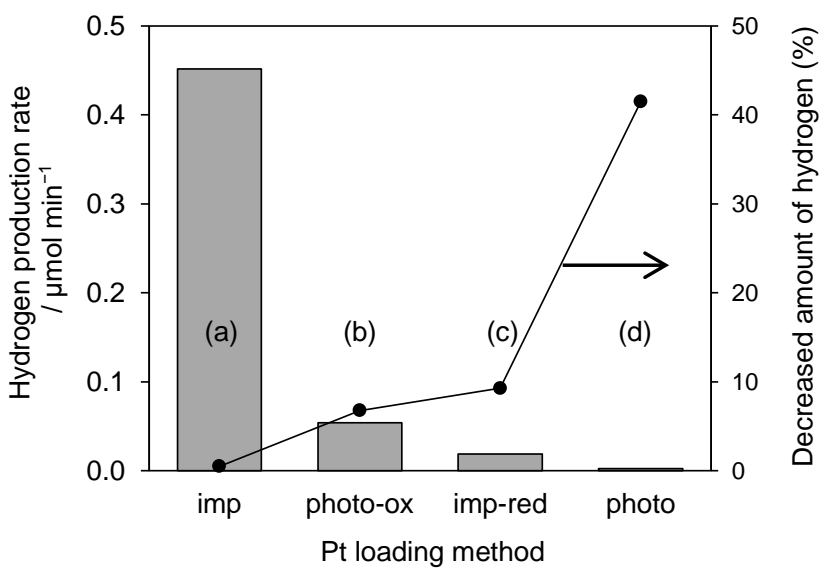

Figure 8. The hydrogen production rate in the photocatalytic water vapour splitting (bars, at the left axis) and the decreased amount of hydrogen in the reaction test for the reverse reaction of water splitting in dark (closed circles, at the right axis) with the $\mathrm{Pt}(0.01) / \mathrm{CTO}$ samples on which Pt cocatalyst was loaded by various methods and post treatments; (a) the Pt(0.01)/CTO-imp sample, (b) the $\mathrm{Pt}(0.01) / C T O-p h o t o-o x$ sample, (c) the $\mathrm{Pt}(0.01) / C T O-i m p-r e d ~ s a m p l e$, and (d) the $\mathrm{Pt}(0.01) / C T O-$ photo sample. The values of the hydrogen production rate and the decreased amount of hydrogen were recorded after $3 \mathrm{~h}$ and $30 \mathrm{~min}$ from the start of the measurement, respectively. The reaction conditions were the same as those for Figures 1 and 2, for the photocatalytic water splitting and the reverse reaction in dark, respectively.

\section{Loading amount of the Pt species}

Figure 9 shows the hydrogen production rates in the photocatalytic water vapor splitting with the bare CTO sample and the various $\mathrm{Pt}(x) /$ CTO catalysts of different Pt loading $x$ wt $\%$. In the series of the $\mathrm{Pt}(x) / C T O$-imp samples prepared by the impregnation method (Figure 9a), the production rate increased with increasing the loading amount and the Pt(0.01)/CTO-imp sample exhibited the highest hydrogen production rate, which was $0.39 \mu \mathrm{mol} / \mathrm{min}^{-1}$, as shown also in Figure 1a. Further increase of the Pt loading amount gave lower production rate, which would be due to the aggregation of the Pt species. Such a volcano plot has been often observed for many metal-loaded photocatalysts.

In the series of the $\mathrm{Pt}(\mathrm{x}) /$ CTO-photo samples prepared by the photodeposition method, the maximum hydrogen production rate was $0.23 \mu \mathrm{mol} / \mathrm{min}^{-1}$ with the $\mathrm{Pt}(0.0025) / C T O-$ photo sample (Figure $9 b)$. Other $\mathrm{Pt}(x) /$ CTO-photo samples with higher or lower $\mathrm{Pt}$ loading amount exhibited lower rates than the $\mathrm{Pt}(0.0025) / \mathrm{CTO}$ photo sample. As mentioned above, when compared at $x=0.01$ $w t \%$, the $\mathrm{Pt}(0.01) / C T O-i m p$ sample exhibited drastically higher production rate than the $\mathrm{Pt}(0.01) / C T O-p h o t o$ sample, and the former showed the highest hydrogen production rate in the present study while the latter produced almost no hydrogen (also, see Figure $1 \mathrm{a}$ and $1 \mathrm{c}$ ).

The reason why the $\mathrm{Pt}(x) /$ CTO-photo sample with the $\mathrm{Pt}$ loading lower than $\mathrm{x}=0.01 \mathrm{wt} \%$ exhibited the hydrogen formation to some extent (Figure 9b) is discussed here. As shown in Figure 
8, the hydrogen production rate becomes higher when the Pt species are less catalytically active for the reverse reaction in dark. The oxidized Pt species shows less catalytic activity for the reverse reaction. If the $\mathrm{Pt}$ nanoparticles of the low loading samples are well dispersed, like as atomically dispersed species, on the $\mathrm{CaTiO}_{3}$ surface, they would be closed to the oxidized state and they would exhibit the higher rate for the production rate of hydrogen and oxygen than the reverse reaction.

The results show that the optimal loading amount of $\mathrm{Pt}$ species were different between the $\mathrm{Pt}(x) /$ CTO-imp and $\mathrm{Pt}(x) /$ CTO-photo samples, which should be discussed here. As mentioned, in the impregnation method, all Pt species $\left(\mathrm{H}_{2} \mathrm{PtCl}_{6}\right)$ in the aqueous solution would be adsorbed on the surface of $\mathrm{CaTiO}_{3}$ during drying up process and react with the surface to make the chemical bonds during the drying and calcination steps. So that, the resulting Pt species would be highly dispersed and stabilized on the surface in the oxidized form, which can contribute to the higher photocatalytic activity. With increasing the $\mathrm{Pt}$ loading amount, the number and the size of the Pt oxide species should increase, which would provide lower photocatalytic activity. On the other hand, in the photodeposition method, a part of the Pt species would be first reduced by the photoexcited electrons to make small Pt metallic clusters on the $\mathrm{CaTiO}_{3}$ surface. Such formed Pt clusters can predominantly receive the photoexcited electrons to reduce the remaining $\mathrm{Pt}$ species on its surface and become larger metallic $\mathrm{Pt}$ nanoparticles. Thus, this method would form larger Pt nanoparticles compared to the impregnation method. The size distributions of the $\mathrm{Pt}(0.2) / \mathrm{CTO}$-imp and $\mathrm{Pt}(0.2) / C T O-$ photo samples are consistent with this explanation (Figure 5), i.e., the latter has relatively larger particles. Thus, the optimal loading amount for the $\mathrm{Pt}(x) /$ CTO-imp samples, $0.01 \mathrm{wt} \%$, was larger than that for the $\mathrm{Pt}(x) / \mathrm{CTO}$-photo samples, $0.0025 \mathrm{wt} \%$.

In our previous study [21], the $\mathrm{Pt}(x) / \mathrm{CaTiO}_{3}$ photocatalysts were prepared by the photodeposition method slightly different condition such as a half time of photoirradiation $(1 \mathrm{~h})$, and the samples with $\mathrm{x}=0.005-0.04 \mathrm{wt} \%$ produced the hydrogen and oxygen in the photocatalytic water vapor splitting, and the $\mathrm{Pt}(0.01) / \mathrm{CaTiO}_{3}$ photocatalysts showed the highest hydrogen production rate such as $0.34 \mu \mathrm{mol} / \mathrm{min}^{-1}$ among them, where the reaction tests were carried out not in the same but similar conditions. This difference on the best loading amount might be related to the difference on the chemical state of the Pt species due to the preparation conditions. The shorter photoirradiation time in the photodeposition might form the more dispersed $\mathrm{Pt}$ nanoparticles.

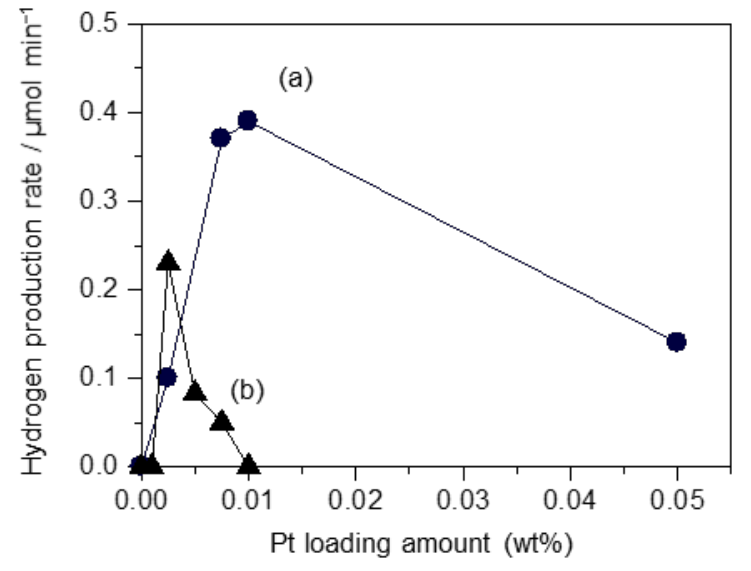

Figure 9. Photocatalytic hydrogen production rate with the samples of various $\mathrm{Pt}$ loading amount, $x$ wt\%, (a) the $\mathrm{Pt}(x) /$ CTO-imp sample and (b) the $\mathrm{Pt}(x) / \mathrm{CTO}$ photo sample. The plot at $x=0$ means the bare СTO sample. The production rate was recorded after $5 \mathrm{~h}$ photoirradiation in the flow of water vapor.

\section{General discussion}

In the present study, a flow of water vapor was introduced to the photoirradiated catalyst bed. The experimental results show that the reverse reaction promoted by the Pt catalyst apparently reduced the progress of the photocatalytic water splitting. Thus, at least in the present reaction conditions using gaseous water, the oxidation state of the Pt cocatalyst is very important, and the property of the $\mathrm{CaTiO}_{3}$ photocatalyst can maintain the oxidized state of the Pt species when they were loaded by the impregnation method. In the present study, it is not experimentally clarified whether these findings are applicable to the liquid phase photocatalytic water splitting or not. Here, it should be discussed in two points as follows.

First point is the separation of the products. In the liquid water, the formed $\mathrm{H}_{2}$ and $\mathrm{O}_{2}$ can escape from the liquid phase to the gas phase and hardly to come back to the photocatalyst surface, so that the effect of the reverse reaction might be low $[5,6]$. However, if the produced gases make bubbles covering the photocatalyst surface including the Pt catalyst, the reverse reaction may take place and reduce the photocatalytic activity. It is notable that even such a case, the Pt/CTO-imp photocatalyst would work efficiently.

Second point is the effect of the reaction temperature. In the present reactor for water vapor, the reaction temperature was increased by photoirradiation. In an aqueous solution, the temperature will be maintained lower such as room temperature due to the large amount of water molecules with the large heat content. Thus, the reaction rate of the catalytic reverse reaction should be lower.

Therefore, from these points of view, the effect of the reverse reaction would be smaller in the water solution. So that, the results clarified in the present study would be more important especially for the photocatalytic water splitting of water vapor. 


\section{Conclusions}

In the present study, it was found that the Pt cocatalyst can function on the $\mathrm{CaTiO}_{3}$ photocatalyst to produce hydrogen and oxygen in the photocatalytic water vapor splitting when it was loaded by the conventional impregnation method around 0.01 wt $\%$ or by the photodeposition method with very low Pt loading amount. The Pt species loaded in the oxidized state are catalytically inactive for the reverse reaction of water splitting, which enables to provide the hydrogen and oxygen in the photocatalytic water vapor splitting.

\section{Experimental Section}

A powder $\mathrm{CaTiO}_{3}$ sample was prepared by a solid-state reaction method reported in the previous study [21]. Starting materials, $\mathrm{CaCO}_{3}$ (Kojundo, 99.99\%) and rutile $\mathrm{TiO}_{2}$ (Kojundo, 99.9\%), were dried at $383 \mathrm{~K}$ for $2 \mathrm{~h}$, weighted in equimolar ratio, and mixed physically by a wet ball-milling method with acetone for $24 \mathrm{~h}$. The obtained powder was dried at $343 \mathrm{~K}$ overnight, calcined on an alumina boat in air at $1273 \mathrm{~K}$ for $10 \mathrm{~h}$, and grained.

Two methods were used for loading Pt cocatalyst on the $\mathrm{CaTiO}_{3}$ sample, i.e., an impregnation method and a photodeposition method. The precursor employed was $\mathrm{H}_{2} \mathrm{PtCl}_{6} \cdot \mathrm{H}_{2} \mathrm{O}$ (Wako 99.9\%) in both methods. The loading amount was in the range of $0.005-0.2 \mathrm{wt} \%$, which values are based on the weight of the starting materials used. In the impregnation method, $2.0 \mathrm{~g}$ of the $\mathrm{CaTiO}_{3}$ powder was impregnated with $50 \mathrm{~mL}$ of an aqueous $\mathrm{H}_{2} \mathrm{PtCl}_{6}$ solution of a desired concentration, stirred in a rotary evaporator at $120 \mathrm{rpm}$ for $30 \mathrm{~min}$, and continuously stirred at $331 \mathrm{~K}$ under evacuation at $120 \mathrm{mbar}$ for $2.5 \mathrm{~h}$, followed by drying at $343 \mathrm{~K}$ in an electric oven overnight. Thus obtained powder was calcined on an alumina boat in air at $773 \mathrm{~K}$ for $6 \mathrm{~h}$. In the photodeposition method, $360 \mathrm{~mL}$ of an aqueous $\mathrm{H}_{2} \mathrm{PtCl}_{6}$ solution containing $2.0 \mathrm{~g}$ of the $\mathrm{CaTiO}_{3}$ powder was magnetically stirred in a $500 \mathrm{~mL}$ beaker covered with a quartz watch glass under photoirradiation by using a $300 \mathrm{~W}$ xenon lamp (PE300BUV) from the upper side for $0.5 \mathrm{~h}$, followed by the addition of $40 \mathrm{~mL}$ methanol and stirring for $1 \mathrm{~h}$ in dark. Then, the suspension was photoirradiated for $2 \mathrm{~h}$ with stirring, and filtered by a membrane filter. The powder was washed by hot water, and dried in the oven overnight at $343 \mathrm{~K}$.

Field emission scanning electron microscopy (SEM) image was recorded by a S-5200 ( $\mathrm{HITACHI})$ with the accelerating voltage of $1.0 \mathrm{kV}$ and the emission current of $10 \mu \mathrm{A}$. Powder $\mathrm{X}$ ray diffraction $(\mathrm{XRD})$ pattern was recorded at room temperature on a MiniFlex-II/AP (Rigaku) using Cu Ka radiation (30 kV, 15 mA) Diffuse reflectance UV-visible spectrum was recorded at room temperature on a $\mathrm{V}-640$ (JASCO) equipped with an integrating sphere covered with $\mathrm{BaSO}_{4}$, where $\mathrm{BaSO}_{4}$ was used as the reference. The Brunauer-Emmett-Teller (BET) specific surface area was calculated from the amount of nitrogen adsorption at 77 $\mathrm{K}$, which was measured by a Monosorb (Quantachrome).

Transmission electron microscope (TEM) images were recorded by a JEOL electron microscope (JEM-2100M, $200 \mathrm{kV}$ ) equipped with a CCD camera (Gatan, erlangshen ES500W). Pt
$\mathrm{L}_{\text {III }}$-edge XAFS (X-ray absorption fine structure) of the Pt-loaded samples were recorded at BL-9C of the Photon Factory at the High Energy Accelerator Research Organization (KEK, Tsukuba Japan) with a Si(111) double-crystal monochromator in a Quick XAFS mode. An ion chamber of the $\mathrm{I}_{0}$ detector was filled with a flow of $\mathrm{N}_{2}(85 \%) / \operatorname{Ar}(15 \%)$. X-ray fluorescence from the sample was recorded by using a Lytle detector filled with an $\operatorname{Ar}(100 \%)$ flow with a Ga filter $(\mu t=6)$. The spectra were analyzed with a REX 2000 software (Rigaku).

The photocatalytic reaction tests for water vapor splitting $\left(2 \mathrm{H}_{2} \mathrm{O} \rightarrow 2 \mathrm{H}_{2}+\mathrm{O}_{2}\right)$ were carried out with a fixed-bed flow reactor. The photocatalyst was pressed at $24 \mathrm{MPa}$ and granulated to the size of $300-600 \mathrm{~mm}$. The quartz cell $\left(60 \times 20 \times 1 \mathrm{~mm}^{3}\right)$ was filled with a mixture of the photocatalyst $(0.8 \mathrm{~g})$ and required amount of quartz granules to fill the cell. The water vapor (1.5\%) with argon carrier was introduced into the reactor at the flow rate of $40 \mathrm{~mL}$ $\mathrm{min}^{-1}$ and the reaction was carried out upon photoirradiation with the $300 \mathrm{~W}$ xenon lamp without using any optical filters, where the light intensity measured in the range of $245 \pm 10 \mathrm{~nm}$ was $10 \mathrm{~mW}$ $\mathrm{cm}^{-2}$. The temperature around the reaction cell rose up to ca. 308 $\mathrm{K}$ upon photoirradiation. The outlet gas was analyzed by on-line gas chromatography with a thermal conductivity detector (Shimadzu, GC-8A, Shincarbon ST, $12 \mathrm{~m}$ ).

The catalytic reaction tests for the reverse reaction of water splitting $\left(2 \mathrm{H}_{2}+\mathrm{O}_{2} \rightarrow 2 \mathrm{H}_{2} \mathrm{O}\right)$ without photoirradiation were carried out in a closed reactor of $62 \mathrm{~mL}$, which was connected to a vacuum system. A part $(0.3 \mathrm{~g})$ of the photocatalyst sample used for the photocatalytic reaction test of water vapor splitting was recovered and introduced in the reactor followed by evacuation at $318 \mathrm{~K}$ for $0.5 \mathrm{~h}$. Since the temperature of the photocatalyst surface during the photocatalytic reaction test mentioned above was presumed to be higher than that of the outside of the reaction cell, the reaction temperature was maintained at $318 \mathrm{~K}$ for this catalytic reverse reaction test. And then hydrogen and oxygen ( 3 and $1.5 \mathrm{kPa}$, respectively) were introduced at $318 \mathrm{~K}$, which was the start of the reaction time. The decrease of the total pressure of the reactant gases with time was monitored by an absolute pressure transducer (MKS Baratron).

\section{Acknowledgements}

The SEM measurements were carried out by the support of Nanofabrication platform at Venture business laboratory (VBL), Nagoya University, under Nanotechnology platform, the Ministry of Education, Culture, Sports, Science and Technology (MEXT), Japan. The Pt $\mathrm{L}_{\mathrm{III}}$-edge XAFS experiments were performed under the approval of the Photon Factory Program Advisory Committee (Proposal No. 2011G575, 2012G586, and 2014G548).

This work was partially supported by a Grant-in-Aid for Scientific Research (C), (No.21560799), a Grant-in-Aid for Scientific Research (B) (No. 25289285), and a Grant-in-Aid for Scientific Research on Innovative Areas "Artificial photosynthesis (AnApple)" (No. 25107515) from the Japan Society for the Promotion of Science (JSPS). 
Keywords: water splitting $\bullet$ hydrogen production $•$ reverse reaction $\bullet$ platinum cocatalyst $\bullet$ calcium titanate photocatalyst

[1] A. Kudo, Y. Miseki, Chem. Soc. Rev. 2009, 38, 253-278.

[2] Y. Inoue, Energy Environ. Sci. 2009, 2, 364-386.

[3] K. Maeda, K. Domen, J. Phys. Chem. Lett. 2010, 1, 2655-2661.

[4] R. Abe, J. Photochem. Photobiol. C 2010, 11, 179-209.

[5] S. Sato, J. M. White, Chem. Phys. Lett. 1980, 72, 83-86.

[6] S. Sato, J. M. White, J. Catal. 1981, 69, 128-139.

[7] Y.H. Li, J. Xing, Z.J. Chen, Z. Li, F. Tian, L.R. Zheng, H.F. Wang, P. Hu, H.J. Zhao, H.G. Yang, Nature Commun. 2013, 4, 2500.

[8] H. Yoshida, S. Kato, K. Hirao, J. Nishimoto, T. Hattori, Chem. Lett. 2007 36, 430-431.

[9] H. Yoshida, K. Hirao, J. Nishimoto, K. Shimura, S. Kato, H. Itoh, T. Hattori, J. Phys. Chem. C 2008, 112, 5542-5551.

[10] K. Shimura, S. Kato, T. Yoshida, H. Itoh, T. Hattori, H. Yoshida, J. Phys. Chem. C 2010, 114, 3493-3503.

[11] K. Shimura, H. Yoshida, Catal. Surv. Asia, 2014, 18, 24-33.

[12] A. Yamamoto, S. Mizuba, Y. Saeki, H. Yoshida, Appl. Catal. A 2016, 521, 125-132.

[13] H. Yoshida, H. Yuzawa, M. Aoki, K. Otake, H. Itoh, T. Hattori, Chem. Commun. 2008, 4634-4636.

[14] H. Yuzawa, T. Mori, H. Itoh, H. Yoshida, J. Phys. Chem. C 2012, 116, 4126-4136.

[15] H. Yuzawa, J. Kumagai, H. Yoshida, J. Phys. Chem. C 2013, 117, $11047-$ 11058.

[16] H. Yuzawa, S. Yoneyama, A. Yamamoto, M. Aoki, K. Otake, H. Itoh, H. Yoshida, Catal. Sci. Technol. 2013, 3, 1739-1749.

[17] E. Wada, A. Tyagi, A. Yamamoto, H. Yoshida, Photochem. Photobio. Sci. 2017, 16, 1744-1748.

[18] A. Tyagi, A. Yamamoto, T. Kato, H. Yoshida, Catal. Sci. Technol. 2017, 7, 2616-2623.

[19] A. Tyagi, A. Yamamoto, M. Yamamoto, T. Yoshida, H. Yoshida, Catal. Sci. Technol. 2018, 8, 2546-2556.

[20] F. Fina, H. Ménard, J.T.S. Irvine, Phys. Chem. Chem. Phys. 2015, 17 13929-13936.

[21] K. Shimura, H. Yoshida, Energy Environ. Sci. 2010, 3, 615-617.

[22] K. Shimura, H. Miyanaga, H. Yoshida, Stud. Surf. Sci. Catal. 2010, 175, 85-92.

[23] H. Yoshida, L. Zhang, M. Sato, T. Morikawa, T. Kajino, T. Sekito, S. Matsumoto, H. Hirata, Catal. Today 2015, 251, 132-139.

[24] A. Anzai, N. Fukuo, A. Yamamoto, H. Yoshida, Catal. Commun. 2017, 100, 134-138.

[25] H. Mizoguchi, K. Ueda, M. Orita, S.C. Moon, K. Kajihara, M. Hirano, H. Hosono, Mater. Res. Bull. 2002, 37, 2401-2406.

[26] H. Yoshida, Y. Yazawa, T. Hattori, Catal. Today 2003, 87, 19-28.

[27] H. Yoshida, S. Nonoyama, Y. Yazawa, T. Hattori, Catal. Today 2010, 153, 156-161.

[28] D. von Bergner, R. Kohlhaas, Z. Anorg. Allg. Chem. 1973, 401, 15-20. 


\section{FULL PAPER}

Active cocatalyst: Pt cocatalyst on $\mathrm{CaTiO}_{3}$ photocatalyst can work to produce hydrogen and oxygen from water vapor when it was prepared by a conventional impregnation method. The Pt species are in the oxidized state on the $\mathrm{CaTiO}_{3}$ surface and catalytically inactive for the reverse reaction of water splitting, which allows to produce hydrogen and oxygen in the photocatalytic water vapor splitting.

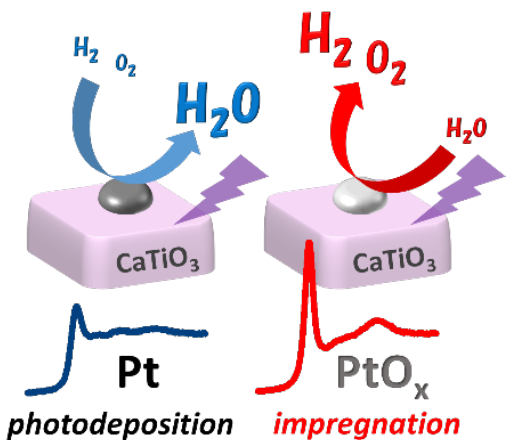

Prof. Hisao Yoshida, * Ryoko Yamada, Prof. Tomoko Yoshida

Page No. - Page No.

Platinum cocatalyst loaded on calcium titanate photocatalyst for water splitting in a flow of water vapor 